\title{
Design of tunable broadband equalizer
}

\author{
Lv Da long ${ }^{1}$, ZHOU Dong fang ${ }^{1}$, Song Cuilian ${ }^{2}$, Zhang Dewei ${ }^{1}$ \\ 1,National Digital Switching System Engineering \& \\ 2,National Institute of Metrology \\ Technological Research Center \\ Zhengzhou P.R.China \\ Beijing, P.R.China \\ ldl2076@yahoo.com.cn \\ songcl@nim.ac.cn
}

\begin{abstract}
Broadband equalizers are widely used in radar systems to compensate for inconsistencies in broadband TWTA's gain response. But few of them are tunable. This paper provides a kind of 6-18GHz coaxial broadband equalizer. The result shows this equalizer is not only small in size, but also convenient to tune. The performance shows it very suitable for broadband use.
\end{abstract}

\section{Keywords-broadband; equalizer; coaxial}

\section{INTRODUCTION}

Equalizers are use to compensate for inconsistencies in TWTA's gain response [1]. Coaxial equalizers are widely used because their high $\mathrm{Q}$ factor, low loss and the ability to tune. We usually think that coaxial equalizers are not suitable in broadband system-for example $6-18 \mathrm{GHz}$ band radar system. The main reason is in broadband system, coaxial resonators may bring two or even more resonating frequencies. Fig 1 shows this character. In broadband system, micrdstrip equalizers are usually used because they are small in size. Many papers have discussed kinds of microstrip equalizers used in broadband system[2] [3] and have good performance. But they also have disadvantage. In many applications, the intersection loss must be tunable while microstrip equalizers are not tunable.

For special applications, broadband equalizers should be tunable according to the curve's varying. How could we do this? This paper realized this through make change with the traditional coaxial equalizers. Compared to traditional coaxial equalizers, the equalizer this paper provided is wider in band and convenient to tune the curve. It is very suitable to be used in broadband equalizer.

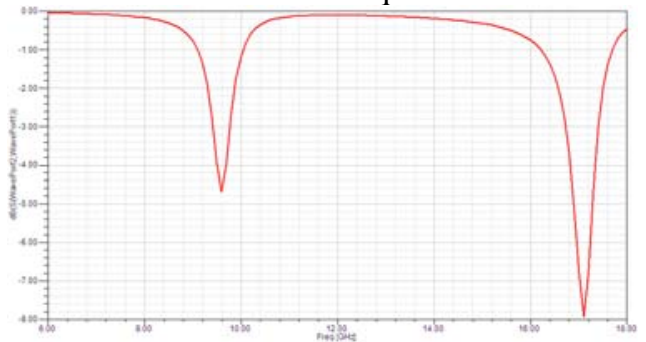

Fig.1 Two resonating frequencies caused by one resonator

\section{STRUCTURE AND ANALYSIS}

Coaxial equalizers usually use $3 \lambda / 4$ or $5 \lambda / 4$ cap acitance loaded resonators. By cascading resonators wit $\mathrm{h}$ different resonating frequencies, different $\mathrm{Q}$ factors $\mathrm{a}$ nd different attenuations, coaxial equalizers can obtain complex $\mathrm{S}_{21}$ curves.
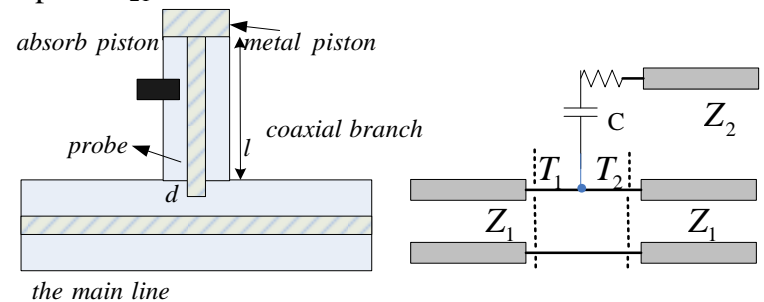

Fig.2 The structure of Small coaxial broadband equalizer and its equivalent

$$
\text { circuit }
$$

The substructure of coaxial equalizer is provided in Fig 2. It is composed of one coaxial cave coupled to the main coaxial transmission line. The resistor is loaded on the bottom of the coaxial cave. In order to calculate the $S$ parameter of the structure, we provided its equivalent circuit as Fig 2, where $\mathrm{C}$ is the couple capacitance.

The characteristic resistance of the main line and branch is $\mathrm{Z} 1$ and $\mathrm{Z} 2$ respectively. The input resistance of branch [4]:

$Z_{a}=R+1 / j \omega C+j Z_{2} \operatorname{tg} \beta l$

$l$ is the length of the branch.

The resonating frequency and the attenuation can be calculated. When it resonates, the follow formula must be right.

$$
\begin{gathered}
1 / \omega C=Z_{2} \operatorname{tg} \beta l \\
L_{\max }=20 \log \left|\frac{2 R}{2 R+Z_{1}}\right|
\end{gathered}
$$

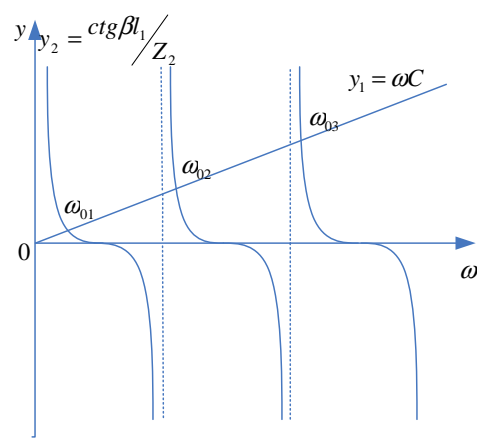

Fig.3 The resonating frequencies when branch length is $l_{1}$ 


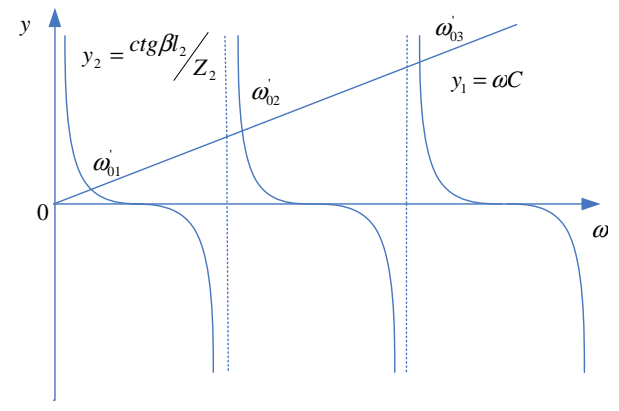

Fig.4 The resonating frequencies when branch length is $l_{2}$

The value of $\mathrm{C}$ and the length of the branch determine the resonating frequency. $\mathrm{C}$ is related to the couple structure. It is almost a fixed value within the bandwidth. We can calculate it by using the formula 2 .

The second rule we can get is from formula 2 which is that the bandwidth of the resonator is determined by the length of the branch. The resonating frequency can be calculated from formulas. In order to understand this more easily, the Fig. 3 and Fig.4 is provided. The length of the branch can affect the bandwidth as well. Fig 3 and Fig 4 show when the length decreases $\left(l_{2}<l_{1}\right)$, the bandwidth will be expanded $\left(\omega_{02}^{\prime}-\omega_{01}^{\prime}>\omega_{02}-\omega_{01}\right)$. So when the bandwidth increases, the problem showed in Fig 1 emerges, it is mostly because the branch is too long.

The third rule is from formula 3 . The attenuation pole of the resonator $L_{\max }$ is mainly determined by the loaded resistor $\mathrm{R}$. The attenuation pole decreased when the resistor increased.

We can see from above expression that in order to form a broadband resonator, the length of the branch must to be much shorter.

This paper uses $\lambda / 4$ capacitance load coaxial resonator. It is mainly for two reasons we choose this length. One reason is to minimize the size, and the other one is to expand the band.

Because the branch is too short to load traditional resistor, the resistor was designed as circle and load at the bottom of the resonator. The structure of coaxial is shown in Fig. 5.

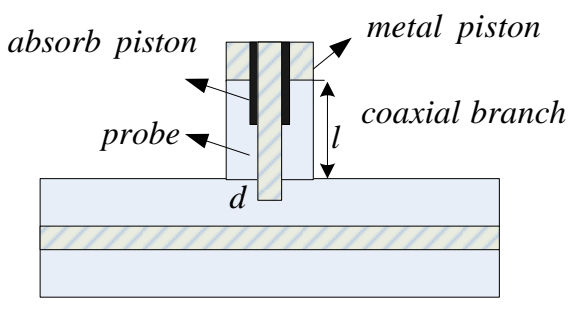

the main line

Fig.5 Structure of coaxial resonator this paper used

\section{SIMULATION}

In this paper, the HFSS simulator from ansoft cooperation is used. The characteristic resistance of the main line is $50 \Omega$. We adjust the transmission curve by changing the length of the resonating cave、 the value of the resistor " $R$ " and the couple capacitance. The length of the resonating cave "l" can be changed by moving the piston. The value of the resistor " $R$ " can be changed by changing the insert length of the absorb piston. And the couple capacitance can be changed by changing the insert length of probe "d". Fig. 6-8 is the results by adjusting "l" 、 " $\mathrm{R}$ " and "d".

We can see three points from the fig.6-8. First, when the length of cave grows, the resonating frequency moves down and the attenuation reduces. Second, when the value of the resistor grows, attenuation reduces while the resonating frequency holds still. Third, when the insert length of the probe grows, attenuation increases and the resonating frequency moves down. All the simulate results are consistent with the analysis result. According to the stimulate result. We can say this resonator is very convenient to adjust.

More we concerned is its additional resonating frequencies. From the HFSS result, we see no other resonating frequency is generated within the band.

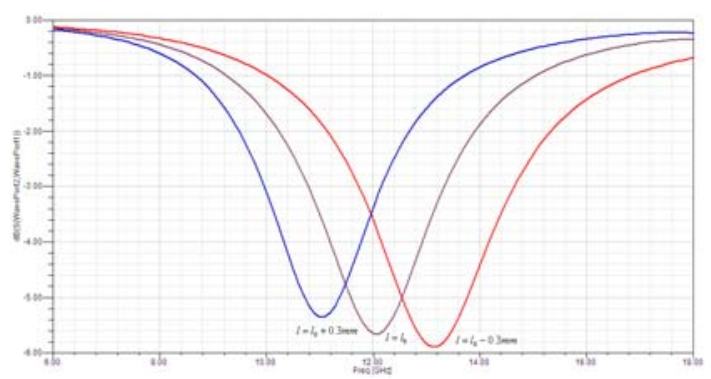

Fig.6 The curve when adjusting “ [ ”

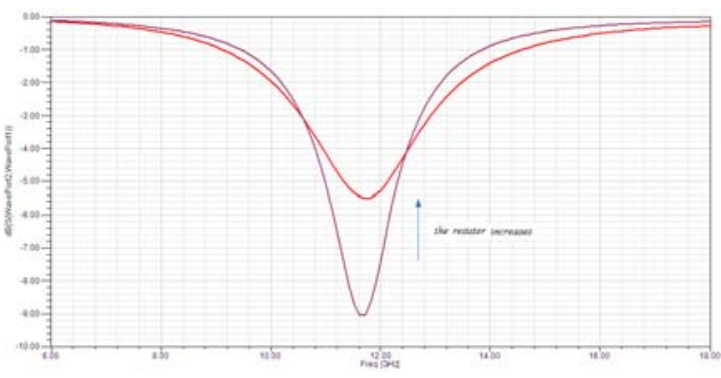

Fig.7 The curve when adjusting resister " $R$ " 


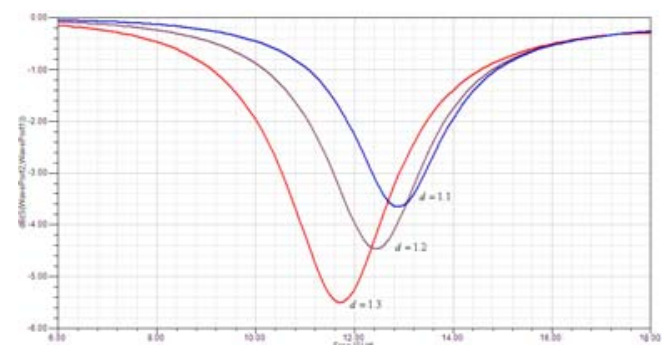

Fig.8 The curve when adjusting “d”

\section{EQUALIZER DESIGN AND MEASUREMENT}

In practical application, the wanted curve is always too complex to be realized with one single resonator. By cascading resonators of different resonating frequencies、 qualities 、 couple coefficients, we can realize complex curves[1][5]. Fig.9 shows the typical curve by cascading resonators with different parameters. The size of this equalizer is only $50 \times 30 \times 20 \mathrm{~mm}^{3}$. It is much smaller than traditional coaxial one.

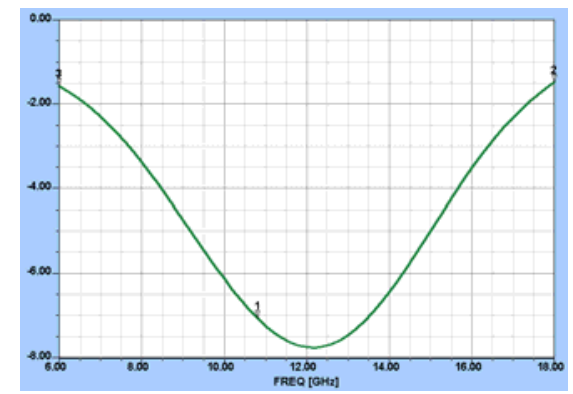

Fig.9 Typical curve

\section{CONCLUSION}

The small coaxial broadband resonator is a novel structure used in broadband equalizers. It is not only small in size but also resonating frequency and attenuation adjustable. It is very suitable for broadband equalizer.

\section{REFERENCES}

[1] Zhang Yi, Niu Zhongxia, Zhou Dongfang, "Design and realization of a novel millimeter wave microstrip equalizer". Journal of infrared and millimeter wave, 2006:393-396

[2] Broadband MIC Equalizers TWTA Output Response. IEEE Design Feature. Oct 1993

[3] A improved branch resonator and it's application in microstrip equalizer designing.Zhao Ying, Zhou Dongfang, Niu Zhongxia, Zhang Dewei.APMC 2005

[4] Liao Cheng-en “Microwave Technology” Xi'an: electronic science and technology university.1985

[5] Ren Jingpu, Zhou Dongfang. "The Substructure analyzing method of interconnect-net”. Proc.ICCEA'99:386-340 